\title{
An Online Mindful Parenting Training for Mothers Raising Toddlers: Assessment of Acceptability, Effectiveness, and Personal Goals
}

\author{
Myrthe G. B. M. Boekhorst ${ }^{1}$ (D) - Lianne P. Hulsbosch ${ }^{1} \cdot$ Ivan Nyklíček ${ }^{1} \cdot$ Viola Spek $^{2} \cdot$ Anna Kastelein $^{3} \cdot$ Susan Bögels $^{3,4}$ • \\ Victor J. M. Pop ${ }^{1}$ Eva S. Potharst ${ }^{3,5}$
}

Accepted: 27 October 2020 / Published online: 9 December 2020

(C) The Author(s) 2020

\begin{abstract}
Objectives Mindful parenting (MP) interventions show promising results, but they mostly target parents (of children) with mental health problems. This study examined an online MP intervention for mothers with toddlers in a population-based sample. Aims were to assess acceptability and effectiveness of the intervention for mothers with and without parental stress, and examine their predetermined personal goals.

Methods The study included 157 mothers with toddlers from the general population of whom 73 reported parental stress. The mothers participated in an 8-week online MP training. Questionnaires were completed at waitlist, pretest, posttest, and follow-up. Results Mothers rated the training positively, although only $23.1 \%$ completed the training. Personal goals were analyzed qualitatively, establishing four different themes: attention, well-being, patience, and balance. Significant improvements in personal goals posttest and follow-up were found (large and very large effect size, respectively). We found no significant improvements from waitlist to pretest for all outcome variables, except personal goals (medium effect size). Mixed-linear model analyses showed significant improvements posttest and follow-up as compared to pretest regarding Self-compassion, Parental overreactivity and Symptoms of anxiety and depression (small to medium effect sizes). There was an effect at posttest for Parenting problems, and for Parental role restriction at follow-up (small effect sizes). Levels of parental stress and theme of personal goal did not influence the effectiveness of the intervention.

Conclusions The current study provides initial evidence that an online MP training could be an easily accessible, inexpensive, and valuable intervention for parents without an indication for a therapist-assisted intervention.

Trial Registration Dutch Trial Register (NTR7401)
\end{abstract}

Keywords Mindful parenting $\cdot$ Online intervention $\cdot$ Personal goals $\cdot$ Parental stress $\cdot$ Population-based sample

Myrthe G. B. M. Boekhorst

m.g.b.m.boekhorst@uvt.nl

1 Center of Research on Psychological and Somatic disorders (CoRPS), Department of Medical and Clinical Psychology, Tilburg University, Warandelaan 2, 5037, AB Tilburg, the Netherlands

2 Department of Applied Psychology, Fontys University of Applied Sciences, Eindhoven, the Netherlands

3 UvA Minds, Academic Outpatient (Child and Adolescent) Treatment Center of the University of Amsterdam, Amsterdam, the Netherlands

4 Developmental Psychology, University of Amsterdam, Amsterdam, the Netherlands

5 Research Institute of Child Development and Education, University of Amsterdam, Amsterdam, the Netherlands
Mindfulness interventions for parents have been widely studied over the last years, showing promising results in reducing levels of parental stress (Burgdorf et al. 2019). Parents can take part in a mindfulness-based stress reduction training (MBSR) (Kabat-Zinn 1990) to practice mindfulness meditation in general. Another type of intervention, mindful parenting (MP) training, is a mindfulness training specifically adapted to the context of parenthood (Bögels and Restifo 2013). MP has been described as a fundamental parenting practice where intentional moment-to-moment non-judgmental awareness is cultivated within the parentchild relationship (Kabat-Zinn and Kabat-Zinn 1997). This non-judgmental moment-to-moment awareness can help parents to become more attentive in the interaction with their children, regarding both (non-)verbal communication and emotional availability. 
In addition, when parents experience increasing levels of parental stress, MP can help them in terms of awareness, acceptance, and the regulation of the stress. As a result, parents will be less likely to impulsively react to their child driven by stress, but rather make a more conscious decision. A MP training specifically aims to help parents cope with, and regulate their parental stress (Bögels et al. 2010; Potharst et al. 2018 b). A systematic review and a recent meta-analysis provided insight in the effectiveness of a MP training and mindfulness interventions for parents of children of different ages and different settings (mental health setting and preventive setting), and showed reduced parental stress and improved child behavioral outcomes (Burgdorf et al. 2019; Townshend et al. 2016). In addition, a recent study on a MP training specifically designed for mothers with a toddler found improvements in parental stress, mother-toddler interactions, and toddler behavioral problems (Potharst et al. 2018b).

Most MP interventions target populations with mental health problems, involving problems in the parents (e.g., mental illness) and/or their children (e.g., behavior or developmental problems). However, little research has assessed the effectiveness of a mindful parenting training in a population-based sample. Potharst et al. (2018a) found that a mindful parenting training was equally effective for parents experiencing elevated levels of parental stress in both a preventative group (parents experiencing parental stress or parenting problems), and a group receiving treatment in an outpatient child and adolescent mental health clinic. Moreover, a more recent study on a mindfulness-based program (MBSR, with adaptations towards parenting) for mothers of children aged 2 to 5 years old in a population-based sample, showed a reduction in parental stress and an improvement in MP (Corthorn 2018). The results of these previously conducted studies suggest that an MP training could also be effective for parents who experience parental stress or parenting problems in a populationbased or preventative setting.

Not only parents with elevated levels of parental stress may benefit from an MP training, it may also be an effective intervention for the general population during early parenthood. In essence, becoming a parent is a joyful experience. However, early parenthood is accompanied by new changes and challenges and can be experienced as overwhelming by parents (Nyström and Öhrling 2004). Toddlerhood is a time that is particularly demanding for parents (Kwon et al. 2013). The challenges arise when toddlers develop more independence along with a stronger will (Edwards and Liu 2002). Due to these challenges, parenting may be especially difficult during toddlerhood.

A MP training can be provided as a group intervention, but these interventions are often aimed at individuals with mental health problems. Despite the possible benefits for mothers with toddlers in the general population, such group interventions are usually not offered to them. Instead, providing MP training by means of the internet can be a good alternative. With the increased access to mobile phone internet, online interventions are easily accessible to most of the population (Andersson 2018; Mohr et al. 2013). Online interventions are convenient and inexpensive, and can solve the barriers that accompany the attendance of group trainings, such as traveling towards and from the group training location and reckoning with the specific time that the training takes place (Lingley-Pottie et al. 2013). A meta-analysis that examined the effectiveness of online parenting programs including 12 studies found promising results on parent and child outcomes, and emphasized the potential of such interventions in increasing parental knowledge and skills (Nieuwboer et al. 2013). Moreover, a meta-analysis of 15 studies on general online mindfulness-based interventions showed positive effects on stress, depression, anxiety, and wellbeing (Spijkerman et al. 2016). Even though these meta-analyses found promising results indicating possible benefits for parents during early parenthood, none of the included studies examined an online MP training. Nonetheless, in a randomized controlled trial (RCT) assessing an online MP training for mothers of 3- to 4-yearold children with elevated levels of parental stress, Potharst et al. (2019) reported a decrease in overreactive parenting, mother-perceived child behavior problems, and maternal symptoms of depression and anxiety, and an increase in selfcompassion. The online version may also be suitable for mothers during toddlerhood who do not necessarily experience profound or heightened levels of parental stress, but who may encounter parenting challenges during this specific period.

Previous research has shown that parents who have a clear (learning) goal in mind, more often meet this desired goal after completing a program or intervention (Darrah et al. 2008; Forsingdal et al. 2014). Therefore, it can be useful for mothers to formulate a personal goal prior to participating in an online MP training. Mapping out what mothers from a general population hope to achieve in such an intervention is also important in order to gain more insight in the specific needs of these mothers and to examine whether an online MP training helps them to achieve their goal. More specifically, it is of importance to gain an understanding of why mothers without elevated levels of parental stress are interested in participating in the online training, and what they personally wish to achieve. The question is whether an online MP training is suitable to achieve these goals and whether the type of personal goal influences the effectiveness of the online MP training.

The current study examined an online MP training for mothers with toddlers in a population-based sample. The aims were to assess (1) the acceptability of the intervention for mothers from a general population, and more specifically, for mothers without elevated parental stress compared to mothers with elevated parental stress; (2) the effectiveness of the combination of goal setting and taking part in the 
intervention for mothers from a general population, and more specifically, for mothers without elevated parental stress compared to mothers with elevated parental stress; (3) categories of predetermined goals set by the participants and whether they made progress with regard to these goals; and (4) whether the effectiveness of the training was dependent on the category of personal goals.

\section{Methods}

\section{Participants}

The current study is part of a large prospective populationbased cohort study following women from the start of their pregnancy onwards: the Holistic Approach to Pregnancy and the first Postpartum Year (HAPPY) study. A detailed protocol of the HAPPY study has previously been described elsewhere (Truijens et al. 2014). A total of 505 women from the HAPPY study participated in a follow-up study until 3.5 years postpartum. All mothers who participated in the follow-up study were invited to participate in the current study when their children were 3 or 4 years of age. Women were included between July 2017 and August 2018. Of the approached women, 172 (34\%) decided to partake in the study, of which 160 (93\%) returned written informed consent and were included for participation. In total, 157 of these women (98\%) formulated a personal goal and were included in the analyses.

Among others, mothers completed the Parental Stress Questionnaire (PSQ) (Vermulst et al. 2012) to assess levels of parental stress at time of inclusion in the current study. The PSQ has three subscales related to parenting: (1) parent child relationship problems, (2) parenting problems, and (3) parental role restriction. Mothers who scored above the cut-off based on the norm-referenced score on at least one of three subscales related to parenting, belonged to the group of participants with "elevated levels of parental stress," and the remaining mothers to the group of participants with "normal levels of parental stress."

Of the 159 women who returned informed consent, two $(1.3 \%)$ failed to return the first questionnaire. This resulted in a sample of $157(98.7 \%)$ mothers that were included in the analyses. We found no differences in sociodemographic characteristics nor levels of parental stress between mothers who did and did not participate in the current study. The demographics and characteristics of the participating mothers are shown in Table 1.

\section{Procedures}

The current study is part of a study with randomized waitlist design, in which both the intervention group and the waitlist group received the intervention, resulting in a pretest, posttest,
Table 1 Characteristics of the participating mothers $(n=157)$

\begin{tabular}{lllll}
\hline & $N$ & $\%$ & Mean (SD) & Range \\
\hline Maternal characteristics & & & & \\
Age & 157 & & $35.6(3.4)$ & $26.2-44.8$ \\
Level of education & & & & \\
Low & 4 & 2.5 & & \\
Medium & 28 & 17.8 & \\
High & 121 & 77.1 & \\
Paid job & 142 & 90.4 & \\
Living with partner & 153 & 97.5 & \\
Elevated levels of & 73 & 53.2 & \\
$\quad$ parental stress & & & \\
Child characteristics & & & \\
Age & & & \\
Gender & & & \\
Girl & 83 & 52.9 & \\
Boy & 72 & 45.9 & \\
Number of children & & & \\
$\quad$ in household & & & \\
One & 21 & 13.4 & \\
Two & 100 & 62.7 & \\
Three & 27 & 17.2 & \\
Four or more & 8 & 5.1 & \\
\hline & & &
\end{tabular}

$S D$, standard deviation; level of education, low: primary education or secondary pre-vocational education; medium: secondary education or vocational education; high: Bachelor or Master's degree

and follow-up measurement for the intervention group, and a waitlist, pretest, and posttest measurement for the waitlist group. We previously reported these randomized waitlist versus intervention group findings in a group of mothers with elevated levels of parental stress, showing that the intervention was more effective than the waitlist period (Potharst et al. 2019). In the current study, we do not analyze the data as a randomized design. Because we examined whether level of parental stress and personal goals were moderating the effectiveness of the training, and we therefore needed to complete interaction analyses, we needed to merge the intervention and waitlist to gain sufficient power. Power analysis was conducted in G-power for sample size calculation. Based on a medium effect size (Eta square $=0.06$ ) for a MANOVA with four time points and power $=0.90$, and an interaction between time and group (four groups), the calculation resulted in a total sample size of 144 women. After merging the groups, our sample size $(N=157)$ was sufficient for these analyses.

The trial is registered in the Dutch Trial Register (NTR7401), and the current study was approved by the Ethics Committee of the University of Amsterdam. After inclusion, women were randomly assigned to either the "intervention group" or the "waitlist group." All participants took part in the same intervention, but their starting time differed 
based on the condition they were assigned to: immediately (intervention group), or after a 10-week waitlist period (waitlist group). For all participants (both groups), the total study duration was 20 weeks, with a 10 -week period between each assessment. Participants completed three assessments during this period, including a waitlist assessment (waitlist group only), pretest assessment, posttest assessment, and follow-up assessment (intervention group only) (see Table 2 for the time points and the different measurements used in the current study). Mothers completed evaluation assessment at posttest to assess the acceptability of the intervention. At all three assessments times, women completed an online questionnaire containing the outcome measures (parental stress, self-compassion, overreactivity in parenting, symptoms of anxiety and depression). Personal goals were formulated in the first assessment and rated during the following two assessments.

Women took part in the online MP training for mothers with toddlers. This training was based on the mindful parenting training (Bögels and Restifo 2013) and the mindful with your toddler training (Potharst et al. 2018b). Adjustments were made to specifically accommodate to the online format and to be applicable for mothers raising toddlers. A mindfulness trainer (EP) and an online-intervention specialist (VS) developed the training. Details on the content of the intervention have been described elsewhere (Potharst et al. 2019). In short, the training consisted of eight weekly online sessions, and each session consisted of the following format: (1) a weekly theme, introduced by a mindfulness trainer in video format; (2) tasks and exercises; (3) additional (written) information about the exercises; and (4) exercises for daily home practice. The training addressed different aspects and domains of mindfulness, including formal meditation (e.g., body scan, sitting meditations, walking meditation), informal meditation and mindful parenting exercises (e.g., visualization exercises).
Mothers had 10 weeks to complete the training. An outline of the MP training has been reported elsewhere (see Potharst et al. 2019). Reflection on personal goals was not only included in the measurements (mothers formulated personal goals as part of the GAS that was administered at the first measurement), but also during the training. The first exercise of week 1 was an "Intention meditation," in which mothers were invited to reflect on and write about why they want to participate in the training, what they would like to learn or change in their lives, and what they are hoping for. In week 5, parents were invited to reflect on the fact that they were halfway in the training, how they had experienced the training thus far, and what they had learned. It was explained to them that it is normal to not be halfway in reaching their goal, as the training has merely focused on awareness, which precedes changes in possible new choices you make as a parent. In week 7, parents reflected on their personal needs, and what actions they wanted to take to better fulfil their personal needs, and in week 8 , parents made a mindfulness plan for after the training.

\section{Measures}

Acceptability To assess acceptability of the training, mothers were asked several questions at the posttest about their experience with the training. Questions were based on the stress reduction program evaluation, developed at the Center for Mindfulness of the University of Massachusetts medical school (Bögels and Restifo 2013). In the current study, this was a 9-item evaluation scale assessing personal changes after the training (see Table 4 for an overview of the evaluation questions). The Cronbach's alpha was .91 at posttest in the current study. Additionally, at posttest, women were asked to grade the training on a scale from 1 to 10 and to indicate their adherence to the training (number of weeks completed,

Table 2 Overview of questionnaires and schedule of assessments during the study period

\begin{tabular}{|c|c|c|c|c|c|c|c|c|}
\hline \multirow[t]{3}{*}{ Aim } & \multirow[t]{3}{*}{ Concept } & \multirow[t]{3}{*}{ Questionnaire } & \multicolumn{6}{|c|}{ Assessments } \\
\hline & & & \multirow{2}{*}{$\begin{array}{l}\text { Waitlist } \\
\text { W }\end{array}$} & \multicolumn{2}{|c|}{ Pretest } & \multicolumn{2}{|c|}{ Posttest } & \multirow{2}{*}{$\begin{array}{l}\text { Follow- } \\
\text { up } \\
\text { I }\end{array}$} \\
\hline & & & & I & $\mathrm{W}$ & I & W & \\
\hline Acceptability & Evaluation intervention & Non-standardized & & & & $\mathrm{X}$ & $\mathrm{X}$ & \\
\hline \multirow[t]{4}{*}{ Effectiveness } & Parental stress & PSQ & $\mathrm{X}$ & $\mathrm{X}$ & $\mathrm{X}$ & $\mathrm{X}$ & $\mathrm{X}$ & $\mathrm{X}$ \\
\hline & Overreactivity in parenting & PS & $\mathrm{X}$ & $\mathrm{X}$ & $\mathrm{X}$ & $\mathrm{X}$ & $\mathrm{X}$ & $\mathrm{X}$ \\
\hline & Self-compassion & SCS-3 & $\mathrm{X}$ & $\mathrm{X}$ & $\mathrm{X}$ & $\mathrm{X}$ & $\mathrm{X}$ & $X$ \\
\hline & Symptoms of anxiety and depression & PHQ4 & $\mathrm{X}$ & $\mathrm{X}$ & $\mathrm{X}$ & $\mathrm{X}$ & $\mathrm{X}$ & $\mathrm{X}$ \\
\hline Personal goals & Personal goals & GAS & $\mathrm{X}$ & $\mathrm{X}$ & $\mathrm{X}$ & $\mathrm{X}$ & $X$ & $X$ \\
\hline
\end{tabular}

There is a 10 -week period between each assessment. $W$, waitlist group; $I$, intervention group; $P S Q$, Parenting Stress Questionnaire; PS, Parenting Scale (Overreactivity subscale); SCS-3, the 3-item version of the Self-Compassion Scale; PHQ4, Patient Health Questionnaire-4; GAS, Goal Attainment Scaling 
number of minutes spent on meditation per week, main reason for not completing the training).

Parental Stress The Dutch version of the 34-item Parenting Stress Questionnaire (PSQ) (Vermulst et al. 2012) was used to assess parental stress. The PSQ can be completed by parents of children between the ages of 0 and 18. Three subscales of the PSQ were used, each assessing a different component of parental stress: (1) parent-child relationship problems (6 items), (2) parenting problems (perceived competence in parenting) (6 items), and (3) parental role restriction (7 items). Mothers were asked to rate items on a four-point Likert scale ( $1=$ not true to $4=$ very true). Higher scores indicate greater levels of parental stress. The PSQ is a valid and reliable instrument to assess parental stress (Veerman et al. 2014; Vermulst et al. 2012). In the current study, the Cronbach's alphas for the subscales were $0.83,0.83$, and 0.80 , respectively.

Overreactivity in Parenting The 10-item overreactivity subscale of the Parenting Scale (PS) (Arnold et al. 1993) was used to assess mothers' overreactive parenting discipline. Overreactivity refers to a parenting discipline that is harsh and authoritative. Each item consists of two statements that are counterparts of one another. For example, "when my child misbehaves," one end of the spectrum is "I usually get into a long argument with my child," and the other "I don't get into an argument." For each item, mothers indicated how they react to a certain situation with their child, on a 7-point scale. Total scores range from 10 to 70 , with higher total scores indicating the use of a more overreactive parenting discipline. The parenting scale has acceptable reliability and validity (Arnold et al. 1993). The Cronbach's alpha for the overreactivity subscale in the current study was .82 .

Self-compassion Self-compassion was assessed using the 3item version of the Self-Compassion Scale (SCS-3, Raes and Neff, unpublished manuscript). The SCS-3 was developed by Raes and Neff and is derived from the Self-Compassion Scale (SCS) (Neff 2003) and the Self-Compassion Scale short-form (SCS-SF) (Raes et al. 2011). The three items all represent a different subscale of the SCS: common humanity, mindfulness, and self-kindness. Mothers rated the three items on a Likert-type scale ranging from 1 to $5(1=$ almost never to 5 = almost always). Total scores range from 3 to 15 , with higher scores indicating higher levels of self-compassion. In the current study, the Cronbach's alpha of this scale was .73.

Symptoms of Anxiety and Depression Mothers completed the four-item Patient Health Questionnaire-4 (PHQ-4) (Kroenke et al. 2009) to assess symptoms of anxiety and depression over the past 2 weeks. The PHQ-4 is developed based on the items from two questionnaires: the General Anxiety Disorder-2
(GAD-2) (Kroenke et al. 2007) and the Patient Health Questionaire-2 (PHQ-2) (Kroenke et al. 2003). Items were rated on a four-point Likert-type scale $(0=$ not at all to $3=$ nearly every day). Total scores ranged from 0 to 12 , with higher scores indicating more symptoms of anxiety and depression. The PHQ-4 has shown to have adequate reliability and validity (Kroenke et al. 2009; Löwe et al. 2010). In the current sample, the Cronbach's alpha of the PHQ-4 was .84.

Mindful Parenting We used the Dutch 10-item version of the Interpersonal Mindfulness in Parenting Scale (IM-P) (Duncan 2007; de Bruin et al. 2014) to assess mindful parenting. In the current study, the Cronbach's alpha for the IM-P was 0.51 and increased to 0.63 after the removal of two items. Considering the weak internal consistency, we did not use the IM-P for analyses in the current study.

Personal Goals Goal Attainment Scaling (GAS) (Kiresuk and Sherman 1968) was used to concretely assess the personal goals that were set by all participating mothers. In the first questionnaire, mothers were asked to formulate an overall goal they wished to have achieved by the end of the intervention. They were also asked to describe this goal more thoroughly and write down what the situation would be like if things were as follows: worse than the current situation ($1)$, according to the current situation (0), a little better than the current situation $(+1)$, a lot better than the current situation $(+2)$, the best possible outcome for the situation $(+3)$ (e.g., 0 $=$ irritable, reacting with irritation at least every morning and evening routine; $-1=$ immediately on edge, reacting with irritation even when there is no hurry and nothing going wrong; $+1=$ more conscious of feelings of irritation before actually reacting with irritation; $+2=$ more conscious of feelings of irritation, and the ability to react calmly either during the morning ritual or the evening ritual; $+3=$ the ability to react calmly during both the morning and evening routine). In the second and third questionnaire, women were shown their previously formulated personal goal and its scales $(-1,0,+1$, +2 , and +3 ), and were asked to indicate what best described their current situation. The GAS has been shown to be a valid and reliable tool to assess personal change (Schlosser 2004; $\mathrm{Vu}$ and Law 2012). In the current study, the content of the personal goals was further examined and it was assessed whether these goals could be divided into different categories.

\section{Data Analyses}

Categorization of Personal Goals Mothers formulated their personal goal (one goal) in the first measurement occasion. Four assessors, who were research assistants with a mindfulness background, assessed the personal goals and were supervised by a mindfulness trainer (EP). Two assessors (assessor 1/assessor 2) independently clustered the personal goals into 
different categories. The assessors specifically analyzed the content of the goals and whether corresponding and common themes could be identified. The personal goal and the explanation of each mother were carefully examined. Assessor 1 and assessor 2 independently provided a collective name for each of those clusters, creating categories for personal goals. After consensus was reached between the first two assessors on the main categories, two new assessors (assessor 3/assessor 4) categorized the personal goals into the different categories that were predefined the first two assessors. Three of the four research assistants were master students (pedagogy or developmental psychology) and interns at an outpatient clinic specialized in mindfulness interventions. They all participated in at least one mindfulness training during this internship. The fourth research assistant was a graduated child psychologist and mindfulness trainer working at the same clinic. The interrater reliability was analyzed with the intraclass correlation coefficient (ICC).

Statistical Analyses Analyses were completed in Statistical Package for the Social Sciences (IBM SPSS version 26.0). Regarding the effectiveness of the training, the repeated measurements at waitlist, pretest, posttest, and follow-up led to a hierarchical dataset. We combined the original $\mathrm{T} 0$ of the intervention group (directly before the start of the intervention) and $\mathrm{T} 1$ of the waitlist group (after the waiting period, and also directly before the start of the intervention) together as a pretest measurement, and the $\mathrm{T} 1$ of the intervention group and $\mathrm{T} 2$ of the waitlist group (both directly after receiving the intervention) as a posttest measurement. Waitlist measurement was only available for the participants that were originally in the waitlist groups, and follow-up assessment was only available for participants that were originally in the intervention group. Mixed model statistics were used to analyze the changes in outcome measures over time. For mixed model analyses, all cases can be included, including those with missing data (Bagiella et al. 2000). Therefore, all participants who completed at least one assessment were included in the analyses. Repeated measurements of time (fixed effects, level 1) nested in participants (level 2) were used to analyze the data. Measures were dummy coded with pretest scores as a reference. In these analyses, pretest was used as a reference so that the outcomes would show waitlist, posttest, and follow-up deviations from pretest. Differences in scores between waitlist and pretest were assessed to examine whether scores remained unchanged prior to the start of the intervention. To assess whether there was a difference in scores between mothers with elevated levels of parental stress and mothers with a normal level of parental stress at posttest and follow-up, interaction variables between time and group were added to the models.

Changes in GAS scores from waitlist to pretest (waitlist group only), pretest to posttest, and posttest to follow-up (intervention group only) were assessed using paired sample $t$ tests. In addition, we examined the changes in GAS scores from pretest to posttest for the entire study sample using GLM repeated measures ANOVA, including interaction variables between time and group of parental stress and time and category of personal goals to assess possible differences in GAS scores per group.

In the mixed model analyses for effectiveness, the time by group interactions at posttest and follow-up were added to assess the possible difference in improvement between participants in different categories of personal goals at the different time points.

\section{Results}

\section{Descriptive Characteristics}

Table 3 shows the mean, standard deviation, and number of participants, for all outcome measures at waitlist, pretest, posttest, and follow-up. At the time of inclusion, 73 (46.5\%) women reported elevated levels of parental stress, 83 (52.9\%) reported normal levels of parental stress, and there was one missing. There were no differences in sociodemographic characteristics between women with and without elevated levels of parental stress. At the time of completing the first questionnaire, mothers with elevated levels of parental stress had higher levels of Parental stress compared to mothers without stress: Parent-child relationship problems $(t(140.35)=-6.11$, $p<0.001)$, Parenting problems $(t(157)=-5.33, p<0.001)$, and Role restriction $(t(157)=-5.39, p<0.001)$, all large effect sizes. Mothers with elevated levels of parental stress also scored higher in overreactivity in parenting $(t(155)=-$ 2.20, $p=0.029$, Cohen's $d=0.35$, small effect size), had lower Self-compassion scores $(t(155)=-2.36, p=0.020$, Cohen's $d=0.37$, small effect size), and reported more symptoms of depression and anxiety $(t(155)=-3.55, p=0.001$, Cohen's $d$ $=0.56$, medium effect size).

\section{Acceptability}

Posttest evaluation by participants is presented in Table 4. Of all the participants who completed posttest assessment, only $31(23.1 \%)$ indicated that they had finished the 8-week online intervention (17.2\% up until week $1,14.2 \%$ week $2,13.4 \%$ week $3,9.7 \%$ week $4,9.7 \%$ week $5,4.5 \%$ week $6,8.2 \%$ week 7 , and $23.1 \%$ week 8 ). The average number of weeks completed was $4.43(\mathrm{SD}=2.61)$. Reasons for not completing the training were as follows: lack of time $(54.8 \%)$, feeling sick (3.8\%), too much stress (4.8\%), feeling better and therefore no need to partake in the training anymore $(1.9 \%)$, not feeling like continuing participation (1.9\%), and quitting for other reasons $(32.7 \%)$. Even though the majority of women did not complete the training, they graded the training with an 
Table 3 Mean and standard deviations for all outcome measures at waitlist, pretest, posttest, and follow-up assessment

\begin{tabular}{lllll}
\hline & $\begin{array}{l}\text { Waitlist } \\
(n=75) \\
\mathrm{M}(\mathrm{SD})\end{array}$ & $\begin{array}{l}\text { Pretest } \\
(n=155) \\
\mathrm{M}(\mathrm{SD})\end{array}$ & $\begin{array}{l}\text { Posttest } \\
(n=135) \\
\mathrm{M}(\mathrm{SD})\end{array}$ & $\begin{array}{l}\text { Follow-up } \\
(n=73) \\
\mathrm{M}(\mathrm{SD})\end{array}$ \\
\hline Parental stress (PSQ) & & & & \\
Parent-child relationship problems & $9.1(2.4)$ & $9.2(2.4)$ & $9.1(2.4)$ & $9.0(2.6)$ \\
Parenting problems & $13.1(2.8)$ & $13.5(2.9)$ & $13.0(2.8)$ & $12.3(3.3)$ \\
Parental role restriction & $11.9(3.2)$ & $11.6(3.0)$ & $11.5(3.0)$ & $10.9(2.6)$ \\
Overreactivity in parenting (PS) & $28.5(8.7)$ & $29.6(8.6)$ & $26.4(7.7)$ & $26.6(7.9)$ \\
Self-compassion (SCS-3) & $12.2(4.0)$ & $12.1(3.9)$ & $13.5(3.5)$ & $13.6(4.0)$ \\
Symptoms of depression and anxiety (PHQ-4) & $2.1(2.1)$ & $2.7(2.7)$ & $2.1(2.5)$ & $1.8(2.4)$ \\
Personal Goals (GAS) & $0.0(0.0)$ & $0.2(0.6)$ & $1.2(0.8)$ & $1.4(1.0)$ \\
\hline
\end{tabular}

Waitlist assessment was completed by the waitlist group only, followed by a 10 -week waitlist period; pretest and posttest assessment were completed by both the waitlist and intervention group; follow-up assessment was completed by the intervention group only. PSQ, Parenting Stress Questionnaire; PS, Parenting Scale; SCS-3, the 3-item version of the Self-Compassion Scale; $P H Q-4$, Patient Health Questionnaire 4; GAS, Goal Attainment Scaling average of 7.0 out of $10(\mathrm{SD}=1.79)$. At follow-up, 23 (31.5\%) mothers continued weekly meditation and 35 (48\%) continued practicing MP after the training, and this did not differ between mothers with and without elevated levels of parental stress. Mothers without elevated levels of parenting stress completed significantly more training weeks $(\mathrm{M}=4.87$, $\mathrm{SD}=2.57$ ) than mothers with elevated levels of parental stress $(\mathrm{M}=3.81, \mathrm{SD}=2.57), \mathrm{t}(131)=2.35, p=0.020$, Cohen's $d=$ 0.40 , small to medium effect size. We found no other differences in posttest evaluation between mothers with and without elevated levels of parental stress.

\section{Effectiveness}

Mixed model analyses assessed the effectiveness of the online MP intervention for all outcome measures during the study period, by examining deviations from pretest assessment (see Table 5 for the results of the mixed model analyses). We found no significant effect of waitlist on the PSQ subscales, indicating no change in scores before the intervention (waitlist to pretest). With regard to the three PSQ subscales, we observed a significant decrease in Parenting problems at posttest (small effect size) and at follow-up in Role restriction (small effect size), but no significant differences in Parent-child relationship problems between pretest assessment and post- or follow-up assessment.

Results also showed that there was no significant effect of waitlist on Overreactivity in parenting and Self-compassion, indicating no change in scores before the intervention (waitlist to pretest). We observed a significant effect of waitlist on Symptoms of depression and anxiety, showing an increase in scores (more symptoms) from waitlist to pretest (small effect size). We also found that, compared to pretest assessment, Overreactivity in parenting, Self-compassion, and Symptoms of anxiety and depression, improved significantly at posttest (small effect sizes), and at follow-up (small to medium effect sizes).

Next, we added interaction variables to the models. Time by group interaction effects at posttest and at follow-up were not significant. This demonstrated that mothers with and without elevated levels of parental stress did not show a difference in change of PSQ subscale scores, Overreactivity in parenting, Self-compassion, and Symptoms of anxiety and depression from pretest to posttest and pretest to follow-up.

\section{Personal Goals}

Four categories of mothers' personal goals were defined. The personal goal of each mother was allocated to one of these categories: (1) attention ( $n=38)$, (2) patience $(n=64)$, (3) balance $(n=31)$, and (4) wellbeing $(n=24)$. Examples of a personal goal for each category were as follows: (1) attention: to become more attentive in the interaction with my children; (2) patience: to maintain patience during conflicts; (3) balance: to find a better balance between work, family, and time for myself; and (4) wellbeing: to lessen the burden of stress and enjoy the moments with my child more consciously. Assessment of interrater reliability showed good reliability $(I C C=0.897(p<.000), 95 \%$ CI $(0.841,0.932))$.

We found no differences in socio-demographic characteristics between mothers in the different groups of personal goals. There were also no differences in the type of personal goal between mothers with and without elevated levels of parental stress $\left(X^{2}(3)=0.88, p=0.830\right)$. Moreover, for each personal goal category, we examined whether mothers differed in baseline scores on the PSQ, PS, SCS-3, and PHQ4 (completed at the same time as formulating their personal goals). The groups significantly 
Table 4 Posttest evaluation of the Online Mindful Parenting Training for mothers with a toddler $(n=134)$

\begin{tabular}{|c|c|c|c|c|}
\hline & & Yes & No & \\
\hline Do you intend to continue practicing formal meditations? & & $70(52.2 \%)$ & $64(47.8 \%)$ & \\
\hline \multirow[t]{2}{*}{ Do you intend to continue practicing mindful parenting? } & & $110(82.1 \%)$ & $24(17.9 \%)$ & \\
\hline & 0 times & 1-2 times & 3-4 times & $>5$ times \\
\hline $\begin{array}{l}\text { From the start of the training, how often did you meditate } \\
\text { per week (do not include practice during the training itself)? }\end{array}$ & $59(44 \%)$ & $54(40.3 \%)$ & $11(8.2 \%)$ & $10(7.5 \%)$ \\
\hline $\begin{array}{l}\text { As a result of the training, has anything changed regarding } \\
\text { the following circumstances: }\end{array}$ & Negative change & No change & Some positive change & Positive change \\
\hline Knowing how to take better care of myself & 0 & $61(45.5 \%)$ & $58(43.3 \%)$ & $15(11.2 \%)$ \\
\hline Actually taking better care of myself & 0 & $77(57.5 \%)$ & $47(35.1 \%)$ & $10(7.5 \%)$ \\
\hline Awareness of stressful parenting situations at the time they are happening & 0 & $34(25.4 \%)$ & $69(51.5 \%)$ & $31(23.1 \%)$ \\
\hline The frequency of parenting stress in the upbringing of my child(ren) & 0 & $70(52.2 \%)$ & $54(40.3 \%)$ & $10(7.5 \%)$ \\
\hline The intensity of stress I experience in the upbringing of my child(ren) & 0 & $69(51.5 \%)$ & $57(42.5 \%)$ & $8(6.0 \%)$ \\
\hline $\begin{array}{l}\text { Dealing with emotions (anger, sadness, anxiety) while parenting / taking } \\
\text { care of my child(ren) }\end{array}$ & 0 & $58(43.3 \%)$ & $64(47.8 \%)$ & $12(9.0 \%)$ \\
\hline The ability to handle stressful parenting situations appropriately & 0 & $56(41.8 \%)$ & $67(50.0 \%)$ & $11(8.2 \%)$ \\
\hline Being content with the relationship and interactions with my child(ren) & $1(0.7 \%)$ & $59(44 \%)$ & $51(38.1 \%)$ & $23(17.2 \%)$ \\
\hline The confidence I have in myself as a parent & 0 & $70(52.2 \%)$ & $41(30.6 \%)$ & $23(17.2 \%)$ \\
\hline
\end{tabular}

The frequencies and percentages $(\%)$ are provided for each item

differed in Parenting problems $(F(3,153)=6.05, p=$ 0.001 ), with post hoc analyses showing that the patience group scored significantly higher (more problems) than the wellbeing $(p=0.016)$ and the balance $(p=0.002)$ groups. We also observed a significant difference in Overreactivity in parenting $(F(3,153)=5.01, p=$ 0.002 ), with post hoc analyses showing that the patience group reported more overreactivity in parenting than the wellbeing $(p=0.012)$ and the balance $(p=0.021)$ groups. Symptoms of depression and anxiety also differed significantly $(F(3,153)=3.05, p=0.030)$, with post hoc analyses showing that the wellbeing group reported higher levels of symptoms than the patience group $(p=0.021)$. There were no differences in Parent-child relationship problems, Role restriction, and Self-compassion.

Results of the paired sample $t$ tests showed a significant improvement in the mothers' personal goal from waitlist $(\mathrm{M}=0.00, \mathrm{SD}=0.00)$ to pretest $(\mathrm{M}=0.44, \mathrm{SD}$ $=0.85) ; t(69)=-4.39, p<0.001$, Cohen's $d=-0.53$, medium effect size. There was a significant improvement form pretest $(\mathrm{M}=0.17, \mathrm{SD}=0.61)$ to posttest $(\mathrm{M}=1.14$, $\mathrm{SD}=0.81) ; t(126)=-12.02, p<0.001$, Cohen's $d=-$ 1.07 , large effect size. There was also a significant improvement form pretest $(\mathrm{M}=0.00, \mathrm{SD}=0.00)$ to followup $(\mathrm{M}=1.40, \mathrm{SD}=0.95) ; t(72)=-12.52, p<0.001$, Cohen's $d=-1.47$, very large effect size. We found similar effects for mothers with and without elevated levels of parental stress at posttest and follow-up (deviation from pretest). Results of GLM repeated measures
ANOVA with an interaction between time and group showed no difference in GAS scores from pretest to posttest between mothers with and without elevated levels of parental stress $(F(1,124)=0.002, p=0.960)$. Paired sample $t$ tests were repeated per category of personal goals (attention, patience, balance, and wellbeing) separately, showing similar results for each category from pretest to posttest (all $p<0.001$, large effect sizes). There was no difference in GAS score from pretest to posttest between categories of personal goals $(F(3,123)=1.14, p$ $=0.335)$. Figure 1 shows mean GAS scores over time, from waitlist to follow-up, per category of personal goals.

We performed mixed model analyses with interaction effects between personal goal category and time at posttest and follow-up to examine possible differences in effectiveness between women in the different categories of personal goals (attention, patience, balance, and wellbeing) during the study period. The interaction between group and time at follow-up was significant for role restriction. The interaction effect showed that the attention group experienced an increase in role restriction (more problems) at follow-up compared to the balance ( $\beta$ $=-0.84, \mathrm{SE}=0.29, t=-2.92, p=0.006)$ and the patience group $(\beta=-0.54, \mathrm{SE}=0.24, t=-2.98, p=$ 0.028 ), which showed a decrease in scores at follow-up compared to pretest. The time by group interaction effects at posttest and at follow-up, for all other outcome variables, were not significant. This demonstrated that the improvements in scores during the study period were not 
dependent on the category of personal goal set by mothers (attention, patience, balance, wellbeing), except for role restriction.

\section{Discussion}

The current study evaluated an online mindful parenting training for mothers raising toddlers in a population-based sample. Because of the fact that only one-third of the invited mothers chose to participate, the sample was select and therefore the results should be interpreted with caution. With regard to the acceptability of the training, results were ambiguous. On the one hand, the participants seemed to be content with the training. They rated the training with 7 points out of 10 . Furthermore, in five out of the nine evaluation areas, more than half of the participants reported improvement (knowing how to take care of themselves, being aware of stress, dealing with stressful events and with difficult emotions, and in being content about the relationship with their child). These outcomes did not differ between the groups of mothers with and without elevated levels of stress. This means that these themes were relevant for both groups, and that there was also room for improvement in the group of mothers without elevated levels of stress. On the other hand, on average only 4 out of eight sessions were completed by the participants. More than half of the women who did not complete the training reported that it was due to lack of time.

Contrary to the other evaluation variables, we found that mothers without elevated levels of parental stress completed a significant higher number of sessions than mothers with elevated stress. The question is how this result can be explained. Self-care practices have been associated with fewer levels of perceived stress (Myers et al. 2012). It could be that mothers with elevated levels of parental stress are not as adequate at taking care of themselves, by continuing to make time to follow the training. Furthermore, the fact that a lack of time was the most common reason for not completing the training seems to point to the possibility that for some women, especially in the group with elevated levels of stress, the self-directed form of the training asked too much from them. In line, a qualitative study evaluating the effects of a self-directed mindfulness training for caregivers reported that especially in stressful periods, participants felt overwhelmed, and this increased difficulties to pursue the training (Stjernswärd and Hansson 2020). A meta-analysis evaluating online mindfulness interventions showed that guided trainings were more effective than self-directed trainings (Spijkerman et al. 2016).

The current study showed that for women who do not experience elevated level of stress, but who do have the wish to become more mindful as a parent, a self-directed (and thus 
Fig. 1 Change in Goal Attainment Scale (GAS) scores (personal goals) throughout the study period from waitlist to follow-up assessment, according to the different categories of personal goals

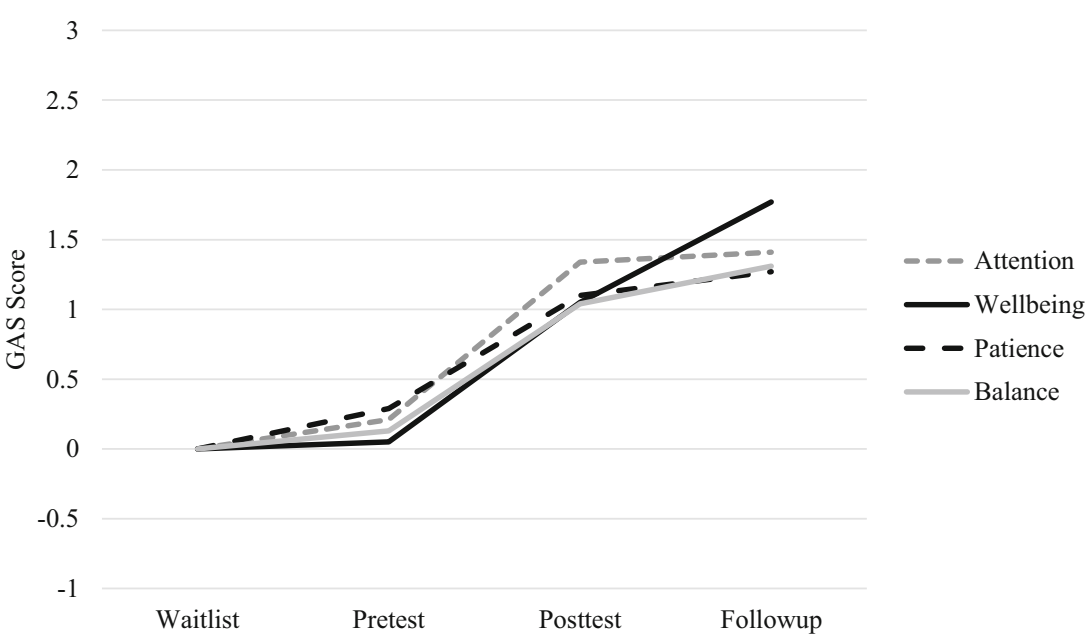

inexpensive) online mindful parenting training may be suitable. Even though mothers received weekly reminders by email in the current study, especially women with elevated levels of parental stress may have preferred or needed online therapist guidance to assist them during the training. Future studies assessing an online MP training should address whether communication with a mindfulness trainer or other professional, or communication with a group, influences the effectiveness and adherence of such interventions.

The results of the current study support the hypotheses that the online MP training may be both acceptable and effective for women without elevated levels of stress. We found positive effects for both groups with regard to parental stress (Parenting problems and Role restriction), Overreactivity in parenting, Self-compassion, and Maternal symptoms of depression and anxiety in a general community sample. However, it may be expected that a group of mothers with elevated levels of parental stress is more likely to benefit from the intervention compared to mothers without elevated levels of parental stress. A meta-analysis on online parenting interventions reported that programs targeting a specific problem seem to be more beneficial than general programs for every parent (Nieuwboer et al. 2013). A possible explanation of the result that mothers with elevated levels of parental stress did not show greater improvements than mothers without elevated levels of parental stress may be that mothers without elevated levels of stress completed more training sessions.

The current study provided initial evidence that mothers who chose to participate in a free online MP training may benefit from such a training, whether they have elevated parental stress levels or not. Meta-analyses that assess mindfulness interventions for people with mental health problems (Goldberg et al. 2018) and healthy people (Khoury et al. 2015) both concluded that mindfulness was moderately effective in pre-post analyses. With regard to mindful parenting, Potharst et al. (2018a) showed that a MP face-to-face group training in a preventative group (parents experiencing parental stress or parenting problems) was as effective as a MP face-toface group training in parents of children with mental health problems.

An explanation for the finding that the online MP training is equally effective for mothers with and without elevated levels of parental stress is that the mothers without elevated levels of parental stress may also experience stress and insecurities in parenting and sometimes respond differently towards their child than they would ideally like. This may especially be the case in the toddlerhood period when mothers face new demanding challenges, such as the growing independence, a strong will or temper tantrums of their child. It has been found that compared to raising younger children, parents of children aged 30-36 months experienced more daily hassles in parenting (Crnic and Booth 1991). Daily parenting hassles are described as "the irritating, frustrating, annoying, and distressing demands that to some degree characterize everyday transactions with the environment" (Crnic and Greenberg 1990). Moreover, these daily hassles, also referred to as minor stressors, are relatively stable over time across early parenthood (Crnic et al. 2005; Winstone et al. 2020). It may be that mothers face parenting hassles daily, regardless of experiencing elevated levels of parental stress, and that an online MP can help mothers cope with this, and with the awareness, acceptance, and the regulation of the associated feelings.

We also found that both mothers with and without elevated levels of parental stress were equally interested to participate in the online MP training. The hypothesis that there is no strict distinction between the mothers with elevated parental stress and the mothers with normal levels of parental stress is illustrated by the examination of the learning goals that the mothers formulated for themselves. The underlying themes of the goals the mothers wished to accomplish after following the training were similar in both groups. This finding can be seen as an illustration of something that is being taught in the MP training, namely the concept of common humanity. 
Common humanity is described as the recognition of the common human experience by acknowledging that all people experience failure, imperfection, and make mistakes (Neff 2016). Common humanity in parenting refers to the understanding that certain difficulties in parenting are universal, and not limited to parents with elevated levels of stress. One such difficulty may be the struggle to find enough time, not only for parenting a child, for work, for a partner and friends, but also for oneself and for personal needs.

The theme of balance reveals this difficulty, which was a theme that appeared from learning goals that mothers formulated. Another example of a universal challenge may be to stay attentive with and available for the child. Parents often recognize this as beneficial for the child as well as oneself on the one hand, but also experience this to be difficult, with all the internal and external distractors that they are often confronted with. This is an example of another theme, attention, which was brought forward by the participating mothers. Interestingly enough, the four themes that were extracted from the personal goals of the participants reflect important aspects of the underlying theoretical framework of mindful parenting that were described by Bögels et al. (2010) and Duncan et al. (2009). The theme patience refers to the concepts of parental reactivity (Bögels et al. 2010) and selfregulation in the parenting relationship (Duncan et al. 2009), the theme balance to self-nourishing attention (Bögels et al. 2010), the theme attention to listening with full attention (Duncan et al. 2009), and well-being to parental stress. This may imply that what intuitively comes up when mothers consider what they would like to learn or improve as a parent, corresponds well with what a MP training has to offer.

This is also endorsed by the outcome that the participants indeed improved on their self-formulated learning goals, irrespective of the category that their goal was attributed to, and irrespective of their level of parental stress. This may indicate that the online MP training is appropriate for mothers with a toddler in general, in achieving their personal goal in parenting. As the different groups of mothers in the current study (with and without elevated level of stress and with different learning goals) seem to benefit from the same intervention, the content of the training seems to be suitable for a broad range of mothers.

\section{Limitations and Future Research}

There are some limitations to take into consideration. An important limitation is related to the design of the study, which only allows for a comparison between the MP training, and no treatment. Therefore, no conclusions can be drawn about the effectiveness of MP as compared to another intervention. As the study participants were not blind to the condition (intervention versus waitlist), both non-specific factors (e.g., taking the time to focus on solving a problem) and expectation bias can lead to an overestimation of the positive effect of the intervention. Future studies should include an active control condition providing parents with another self-directed intervention with non-specific therapeutic characteristics but without the specific mindful parenting mechanisms. Furthermore, due to the fact that all measures were common in the way they were administrated and completed (self-report, online, multiple-item scales (except for the GAS) and in one survey per measurement), our results may have been influenced by common methods bias (Podsakoff et al. 2003). Potential causes for common method bias in the current study are social desirability (especially at posttest) and transient mood state. A consequence of common methods bias in the current study may have been a higher correlation between the outcome measures, which may have resulted in a higher number of outcome measures showing significant improvement at posttest. Another limitation is that in comparison to the general Dutch population, the current sample was predominantly white, highly educated and the majority had a partner. This may limit generalization of the results of the current study. Mindfulness interventions have shown to be appropriate for different cultural populations and ethnic minorities, but adaptations to heighten cultural relevance are recommended (Hall et al. 2011; Woods-Giscombé and Gaylord 2014). Additionally, with regard to mindful parenting, research showed that the factor structure of the Interpersonal in Mindfulness in Parenting scale (IM-P) differs per country and/or culture, possibly reflecting the cultural differences in parenting styles and norms (de Bruin et al. 2014; Pan et al. 2019). These findings could indicate that MP intervention may also require slight adaptations to align with culturally relevant parenting norms. Future studies should examine the effectiveness and acceptability of an online MP training in a more diverse sample of mothers and take into consideration the cultural values in parenting for different samples. In the current study, mothers completed the 10 -item version of the IM-P throughout the study-period, but its internal consistency was weak and, therefore, we were not able to use this tool. Consequently, we could not assess whether the positive effects of the training were due to an improvement in levels of mindful parenting. Next, adherence to the training was low, with only a small percentage of the mothers completing the entire 8-week MP training. Another limitation of the current study is that only $34 \%$ of the invited participants agreed to participate. This could possibly indicate sampling bias as it may be that mothers with an affiliation or greater intention to practice mindfulness were more likely to participate. This may limit generalization of the results. Moreover, the current sample only consisted of mothers while fathers were not included. Several studies have demonstrated that mothers experience more stress on different domains of parental stress compared to fathers (Hildingsson and Thomas 2014; Widarsson et al. 2013) and mothers show higher levels of mindful 
parenting (Moreira and Canavarro 2015). Future studies should consider these differences and include fathers in studies examining an online MP training.

Author Contributions MB contributed to data collection and data analysis and wrote the paper. LH contributed to data analysis and wrote parts of the manuscript. IN and VS contributed to the development of the training and to the study design. AK contributed to the analysis of qualitative data. SB contributed to the development of the training and to the study design. $\mathrm{VP}$ is the principal investigator and acquired funding, contributed to the study design, and monitored the process of data collection and analysis. EP contributed to the development of the training and study design, and monitored the process of data analyses and writing of the study and collaborating in the writing and editing of the manuscript. All authors contributed to the final version of the manuscript.

Funding This study was funded by Stichting de Weijerhorst.

\section{Compliance with Ethical Standards}

Conflict of Interest The authors declare that they have no conflict of interest.

Ethics Statement Ethical approval: all procedures performed in studies involving human participants were in accordance with the ethical standards of the institutional and/or national research committee (Ethics Committee of the University of Amsterdam) and with the 1964 Helsinki declaration and its later amendments or comparable ethical standards.

Informed Consent Informed consent was obtained from all individual participants included in the study.

Open Access This article is licensed under a Creative Commons Attribution 4.0 International License, which permits use, sharing, adaptation, distribution and reproduction in any medium or format, as long as you give appropriate credit to the original author(s) and the source, provide a link to the Creative Commons licence, and indicate if changes were made. The images or other third party material in this article are included in the article's Creative Commons licence, unless indicated otherwise in a credit line to the material. If material is not included in the article's Creative Commons licence and your intended use is not permitted by statutory regulation or exceeds the permitted use, you will need to obtain permission directly from the copyright holder. To view a copy of this licence, visit http://creativecommons.org/licenses/by/4.0/.

\section{References}

Andersson, G. (2018). Internet interventions: past, present and future. Internet Interventions, 12, 181-188.

Arnold, D. S., O’Leary, S. G., Wolff, L. S., \& Acker, M. M. (1993). The Parenting Scale: a measure of dysfunctional parenting in discipline situations. Psychological Assessment, 5(2), 137-144.

Bagiella, E., Sloan, R. P., \& Heitjan, D. F. (2000). Mixed-effects models in psychophysiology. Psychophysiology, 37(1), 13-20.

Bögels, S. M., \& Restifo, K. (2013). Mindful parenting: a guide for mental health practitioners. New York: Springer Science \& Business Media.

Bögels, S. M., Lehtonen, A., \& Restifo, K. (2010). Mindful parenting in mental health care. Mindfulness, 1(2), 107-120.
Burgdorf, V., Szabó, M., \& Abbott, M. J. (2019). The effect of mindfulness interventions for parents on parenting stress and youth psychological outcomes: a systematic review and meta-analysis. Frontiers in Psychology, 10. https://doi.org/10.3389/fpsyg.2019.01336.

Corthorn, C. (2018). Benefits of mindfulness for parenting in mothers of preschoolers in Chile. Frontiers in Psychology, 9. https://doi.org/10. 3389/fpsyg.2018.01443.

Crnic, K. A., \& Booth, C. L. (1991). Mothers' and fathers' perceptions of daily hassles of parenting across early childhood. Journal of Marriage and the Family, 53(4), 1042.

Crnic, K. A., \& Greenberg, M. T. (1990). Minor parenting stresses with young children. Child Development, 61, 1628-1637.

Crnic, K. A., Gaze, C., \& Hoffman, C. (2005). Cumulative parenting stress across the preschool period: relations to maternal parenting and child behaviour at age 5. Infant and Child Development, 14(2), 117-132.

Darrah, J., Wiart, L., \& Magill-Evans, J. (2008). Do therapists' goals and interventions for children with cerebral palsy reflect principles in contemporary literature? Pediatric Physical Therapy, 20(4), 334 339.

de Bruin, E. I., Zijlstra, B. J. H., Geurtzen, N., van Zundert, R. M. P., van de Weijer-Bergsma, E., Hartman, E. E., Nieuwesteeg, A. M., Duncan, L. G., \& Bögels, S. M. (2014). Mindful parenting assessed further: psychometric properties of the Dutch version of the Interpersonal Mindfulness in Parenting scale (IM-P). Mindfulness, $5(2), 200-212$.

Duncan, L. G. (2007). Assessment of mindful parenting among parents of early adolescents: development and validation of the Interpersonal Mindfulness in Parenting Scale [Doctoral dissertation]. The Pennsylvania State University.

Duncan, L. G., Coatsworth, J. D., \& Greenberg, M. T. (2009). A model of mindful parenting: implications for parent-child relationships and prevention research. Clinical Child and Family Psychology Review, 12(3), 255-270.

Edwards, C. P., \& Liu, W. L. (2002). Parenting toddlers. In M. H. Bornstein (Ed.), Handbook of parenting: children and parenting (pp. 45-71). Mahwah: Lawrence Erlbaum.

Forsingdal, S., St John, W., Miller, V., Harvey, A., \& Wearne, P. (2014). Goal setting with mothers in child development services. Child: Care, Health and Development, 40(4), 587-596.

Goldberg, S. B., Tucker, R. P., Greene, P. A., Davidson, R. J., Wampold, B. E., Kearney, D. J., \& Simpson, T. L. (2018). Mindfulness-based interventions for psychiatric disorders: a systematic review and meta-analysis. Clinical Psychology Review, 59(12), 52-60.

Hall, G. C. N., Hong, J. J., Zane, N. W. S., \& Meyer, O. L. (2011). Culturally competent treatments for Asian Americans: the relevance of mindfulness and acceptance-based psychotherapies. Clinical Psychology: Science and Practice, 18(3), 215-231.

Hildingsson, I., \& Thomas, J. (2014). Parental stress in mothers and fathers one year after birth. Journal of Reproductive and Infant Psychology, 32(1), 41-56.

Kabat-Zinn, J. (1990). Full catastrophe living: using the wisdom of your body and mind to face stress, pain, and illness. Delta Trade Paperbacks.

Kabat-Zinn, M., \& Kabat-Zinn, J. (1997). Everyday blessings: the inner work of mindful parenting. New York: Hyperion.

Khoury, B., Sharma, M., Rush, S. E., \& Fournier, C. (2015). Mindfulness-based stress reduction for healthy individuals: a metaanalysis. Journal of Psychosomatic Research, 78(6), 519-528.

Kiresuk, T. J., \& Sherman, R. E. (1968). Goal attainment scaling: a general method for evaluating comprehensive community mental health programs. Community Mental Health Journal, 4(6), 443453.

Kroenke, K., Spitzer, R. L., \& Williams, J. B. W. (2003). The Patient Health Questionnaire-2. Medical Care, 41(11), 1284-1292. 
Kroenke, K., Spitzer, R. L., Williams, J. B. W., Monahan, P. O., \& Löwe, B. (2007). Anxiety disorders in primary care: prevalence, impairment, comorbidity, and detection. Annals of Internal Medicine, 146(5), 317-325.

Kroenke, K., Spitzer, R. L., Williams, J. B. W., \& Löwe, B. (2009). An ultra-brief screening scale for nxiety and depression: the PHQ 4 . Psychosomatics, 50(6), 613-621.

Kwon, K. A., Han, S., Jeon, H. J., \& Bingham, G. E. (2013). Mothers' and fathers' parenting challenges, strategies, and resources in toddlerhood. Early Child Development and Care, 183(3-4), 415-429.

Lingley-Pottie, P., McGrath, P. J., \& Andreou, P. (2013). Barriers to mental health care. Advances in Nursing Science, 36(1), 51-61.

Löwe, B., Wahl, I., Rose, M., Spitzer, C., Glaesmer, H., Wingenfeld, K., et al. (2010). A 4-item measure of depression and anxiety: validation and standardization of the Patient Health Questionnaire-4 (PHQ-4) in the general population. Journal of Affective Disorders, 122(1-2), $86-95$.

Mohr, D. C., Burns, M. N., Schueller, S. M., Clarke, G., \& Klinkman, M. (2013). Behavioral intervention technologies: evidence review and recommendations for future research in mental health. General Hospital Psychiatry, 35(4), 332-338.

Moreira, H., \& Canavarro, M. C. (2015). Individual and gender differences in mindful parenting: the role of attachment and caregiving representations. Personality and Individual Differences, 87, 13-19.

Myers, S. B., Sweeney, A. C., Popick, V., Wesley, K., Bordfeld, A., \& Fingerhut, R. (2012). Self-care practices and perceived stress levels among psychology graduate students. Training and Education in Professional Psychology, 6(1), 55-66.

Neff, K. D. (2003). The development and validation of a scale to measure self-compassion. Self and Identity, 2(3), 223-250.

Neff, K. D. (2016). The Self-Compassion Scale is a valid and theoretically coherent measure of self-compassion. Mindfulness, 7(1), 264 274.

Nieuwboer, C. C., Fukkink, R. G., \& Hermanns, J. M. A. (2013). Online programs as tools to improve parenting: a meta-analytic review. Children and Youth Services Review, 35(11), 1823-1829.

Nyström, K., \& Öhrling, K. (2004). Parenthood experiences during the child's first year: literature review. Journal of Advanced Nursing, 46(3), 319-330.

Pan, J., Liang, Y., Zhou, H., \& Wang, Y. (2019). Mindful parenting assessed in mainland China: psychometric properties of the Chinese version of the Interpersonal Mindfulness in Parenting scale. Mindfulness, 10(8), 1629-1641.

Podsakoff, P. M., MacKenzie, S. B., Lee, J. Y., \& Podsakoff, N. P. (2003). Common method biases in behavioral research: a critical review of the literature and recommended remedies. Journal of Applied Psychology, 88(5), 879-903.

Potharst, E. S., Baartmans, J. M. D., \& Bögels, S. M. (2018a). Mindful parenting training in a clinical versus non-clinical setting: an explorative study. Mindfulness. Advance of Print. https://doi.org/10.1007/ s12671-018-1021-1.

Potharst, E. S., Zeegers, M., \& Bögels, S. M. (2018b). Mindful with your toddler group training: Feasibility, acceptability, and effects on subjective and objective measures. Mindfulness. Advance of Print. https://doi.org/10.1007/s12671-018-1073-2.

Potharst, E. S., Boekhorst, M. G. B. M., Cuijlits, I., van Broekhoven, K. E. M., Jacobs, A., Spek, V., Nyklíček, I., Bögels, S. M., \& Pop, V. J. M. (2019). A randomized control trial evaluating an online mindful parenting training for mothers with elevated parental stress. Frontiers in Psychology, 10. https://doi.org/10.3389/fpsyg.2019. 01550.

Raes, F., Pommier, E., Neff, K. D., \& Van Gucht, D. (2011). Construction and factorial validation of a short form of the SelfCompassion Scale. Clinical Psychology \& Psychotherapy, 18(3), 250-255.

Schlosser, R. W. (2004). Goal attainment scaling as a clinical measurement technique in communication disorders: a critical review. Journal of Communication Disorders, 37(3), 217-239.

Spijkerman, M. P. J., Pots, W. T. M., \& Bohlmeijer, E. T. (2016). Effectiveness of online mindfulness-based interventions in improving mental health: a review and meta-analysis of randomised controlled trials. Clinical Psychology Review, 45, 102-114.

Stjernswärd, S., \& Hansson, L. (2020). A qualitative study of caregivers' experiences, motivation and challenges using a web-based mindfulness intervention. Community Mental Health Journal, 56(3), 416425.

Townshend, K., Jordan, Z., Stephenson, M., \& Tsey, K. (2016). The effectiveness of mindful parenting programs in promoting parents' and children's wellbeing: a systematic review. JBI Database of Systematic Reviews and Implementation Reports, 14(3), 139-180.

Truijens, S. E. M., Meems, M., Kuppens, S. M. I., Broeren, M. A. C., Nabbe, K. C. A. M., Wijnen, H. A., Oei, S. G., van Son, M. J. M., \& Pop, V. J. M. (2014). The HAPPY study (Holistic Approach to Pregnancy and the first Postpartum Year): design of a large prospective cohort study. BMC Pregnancy and Childbirth, 14(1), 1-12. https://doi.org/10.1186/1471-2393-14-312.

Veerman, J. W., Kroes, G., De Meyer, R. E., Nguyen, L. M., \& Vermulst, A. A. (2014). Opvoedingsbelasting in kaart gebracht. Een kennismaking met de Opvoedingsbelastingvragenlijst (OBVL) [Parenting stress mapped out. An introduction to the Parenting Stress Questionnaire (PSQ)]. JGZ Tijdschrift voor Jeugdgezondheidszorg, 46(3), 51-55.

Vermulst, A., Kroes, G., De Meyer, R., Nguyen, L., \& Veerman, J. W. (2012). Opvoedingsbelastingvragenlijst (OBVL). Handleiding. [Parenting Stress Questionnaire (PSQ). Manual]. Nijmegen: Praktikon.

Vu, M., \& Law, A. V. (2012). Goal-attainment scaling: a review and applications to pharmacy practice. Research in Social and Administrative Pharmacy, 8(2), 102-121.

Widarsson, M., Engström, G., Rosenblad, A., Kerstis, B., Edlund, B., \& Lundberg, P. (2013). Parental stress in early parenthood among mothers and fathers in Sweden. Scandinavian Journal of Caring Sciences, 27(4), 839-847.

Winstone, L. K., Curci, S. G., \& Crnic, K. A. (2020). Pathways to maternal and child well-being: Stability and transaction across toddlerhood. Parenting, 1-23. Advance of print. https://doi.org/10.1080/ 15295192.2019.1701933.

Woods-Giscombé, C. L., \& Gaylord, S. A. (2014). The cultural relevance of mindfulness meditation as a health intervention for African Americans: implications for reducing stress-related health disparities. Journal of Holistic Nursing, 32(3), 147-160.

Publisher's Note Springer Nature remains neutral with regard to jurisdictional claims in published maps and institutional affiliations. 